\title{
Adsorption of atrazine from aqueous solution using magnetic grapheme oxide
}

\author{
Xiaojun Zhang, Xiaoguo Ma*, Weiheng Shu and Junbiao Peng \\ School of Environmental Science and Engineering, Guangdong University of Technology, \\ Guangzhou 510006, China \\ xgma@21cn.com
}

Keywords: Magnetic graphene oxide; atrazine; adsorption.

Abstract. Magnetic graphene oxide (MGO) was prepared, and characterized using Fourier transform infrared spectroscopy and transmission electronic microscopy. The MGO was used as the sorbent for adsorption of atrazine, and the adsorption performance was investigated under various conditions. Results indicated that the adsorption equilibrium was achieved within $2 \mathrm{~h}$, the adsorption capacity was $7.52 \mathrm{mg} / \mathrm{g}$, and the adsorption process could be well described by Freundlich isotherm model and pseudo-second-order kinetic model. The hexatomic ring as well as carboxyl, carbonyl, epoxy and hydroxy groups on MGO were the main adsorption sites, and there are $\pi-\pi$ interaction and hydrogen -bond interaction of MGO with atrazine. The experimental results showed MGO could be a potential adsorbent for removal of atrazine from aqueous solution.

\section{Introduction}

Atrazine (2-chloro-4-ethylamino-6-isopropylamino-1, 3, 5-triazine) is the most widely used herbicide, which is mainly used to remove broadleaf and grassy weeds in corn or crops. Atrazine is now considered as a potential contamination which can cause cancer and do harm to endocrine system of human [1]. Nowadays, atrazine has been detected in various environmental matrices. Consequently, it is necessary to remove atrazine from wastewater. Adsorption is one of the most important methods for separation of metal ion and organic pollutants from aqueous solutions [2-4], due to its high efficiency and easy operation. Obviously, sorbent is the key factor of this technique.

Graphene oxide (GO) is the oxidized derivative of graphene, which possesses an ultrahigh-specific surface area, superior chemical property and excellent thermal stability. Therefore, GO has great promise for use as adsorbent materials [5].

Magnetically separation technology provides an easy and rapid way for removal of magnetic particles from solution by applying an appropriate magnetic field [6]. Thus, magnetic adsorbent can be easily isolated from matrix solutions after adsorption of target compounds.

In this work, magnetic (MGO) was prepared and characterized, and was applied for the removal of atrazine from aqueous solution. The adsorption behaviors of atrazine on the MGO were investigated and evaluated.

\section{Materials and methods}

\section{Reagents}

Atrazine was obtained from Aladdin Reagent (Shanghai) Co., Ltd (Shanghai, China).Nature graphite powder $(99.95 \%)$ was purchased from Sinopharm Chemical Reagent Co., Ltd (Shanghai, China). Other chemicals and materials were purchased from Tianjin Damao Chemical Reagent Factory (Tianjin, China).

Instruments

A 7890A-5975C GC-MS system (Agilent, USA) using a HP-5MS capillary columnwas utilized for separation and analysis. 


\section{Preparation of $M G O$}

Graphite oxide (GO) was prepared from nature graphite powders by a modified Hummers method [7]. Magnetic graphite oxide (MGO) was synthesized by a one-pot solvothermal reaction [8].

\section{Results and discussion}

\section{Characterization of $M G O$}

The FT-IR spectrum of the prepared MGO is shown in Fig.1. As can be seen, the peak at $571 \mathrm{~cm}^{-1}$ was related to $\mathrm{Fe}-\mathrm{O}$ stretching vibrations. The peak at $1398 \mathrm{~cm}^{-1}$ was assigned to $\mathrm{C}=\mathrm{C}$ from unoxidized $\mathrm{sp}^{2} \mathrm{CC}$ bonds. The peak at $1576 \mathrm{~cm}^{-1}$ corresponded to $v(\mathrm{C}=\mathrm{O})$ of $-\mathrm{COOH}$ on the $\mathrm{GO}$ after coating with $\mathrm{Fe}_{3} \mathrm{O}_{4}$. The peaks at $3385 \mathrm{~cm}^{-1}$ was attributed to $\mathrm{O}-\mathrm{H}$ stretching vibrations.

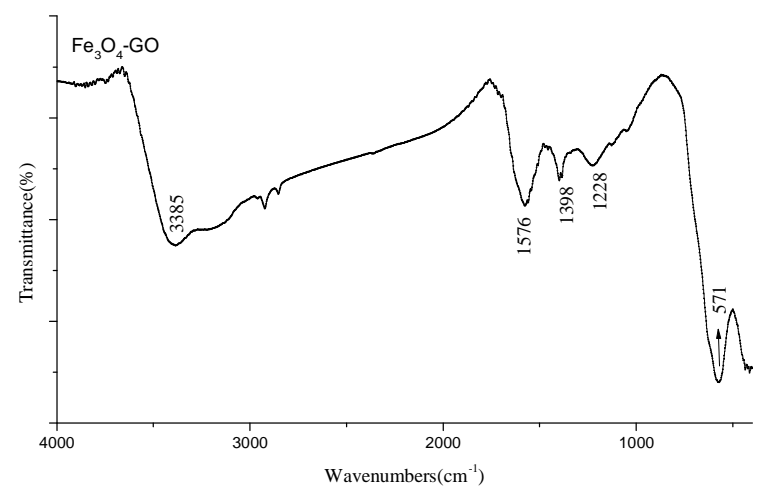

Fig.1 FTIR spectrum of MGO.

From the TEM observation (Fig.2), it was found that the $\mathrm{Fe}_{3} \mathrm{O}_{4}$ particles on $\mathrm{GO}$ were almost regular in shape with an average diameter of around $20 \mathrm{~nm}$.

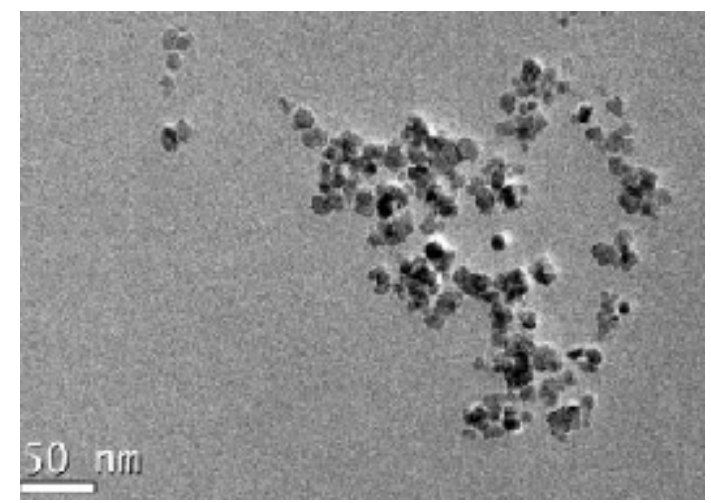

Fig.2 TEM image of MGO.

The magnetic property of the MGO was tested with a vibrating sample magnetometer. The greatest saturation magnetization was $29.8 \mathrm{emu} / \mathrm{g}$, and both the remanence and coercivity were near zero, suggesting that the MGOwere superparamagnetic.

The adsorption of atrazine on the MGO was examined using batch experiments and conducted in duplicate. The adsorption capacities varied with initial atrazine concentrations (Fig.3). Langmuir and Freundlich isotherms were used to describe the adsorption behaviors. Freundlich isotherm mode fitted the experimental data better $\left(R^{2}=0.9987\right)$ than the Langmuir model $\left(R^{2}=0.8703\right)$, suggesting that the adsorption of atrazine onto the MGO followed the Freundlich isotherm (Fig.4). The 
maximum adsorption capacity was $7.5 \mathrm{mg} / \mathrm{g}$ at an initial atrazine concentration of $10 \mathrm{mg} / \mathrm{L}$. Some important thermodynamic parameters variations including standard Gibbs free energy $\left(\triangle G^{0}\right)$, enthalpy $\left(\triangle H^{0}\right)$ and entropy change $\left(\triangle S^{0}\right)$ for the adsorption process were obtained. $\triangle G^{0}$ has negative value $(-12.5 \mathrm{KJ} / \mathrm{mol})$, indicating that the adsorption was spontaneous. On the other hand, the positive value of $\triangle H^{0}(50.9 \mathrm{KJ} / \mathrm{mol})$ suggested an endothermic nature of atrazine adsorption.

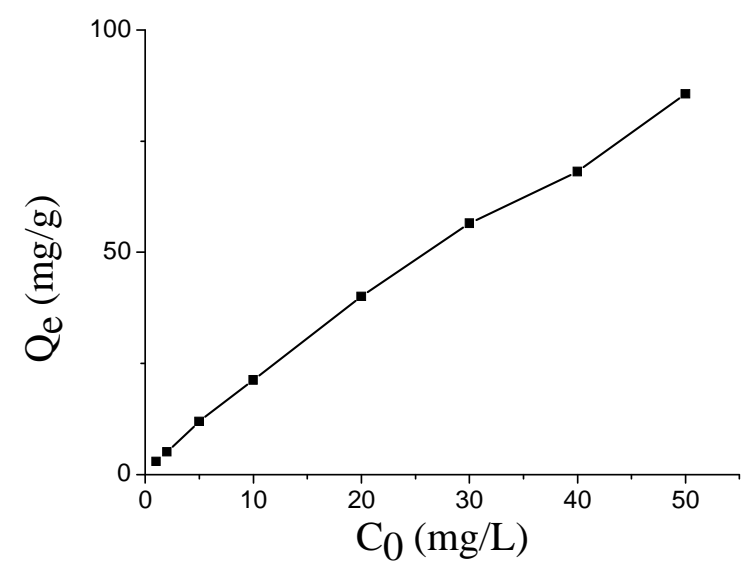

Fig.3 Atrazine adsorption on MGO at different initial concentrations.

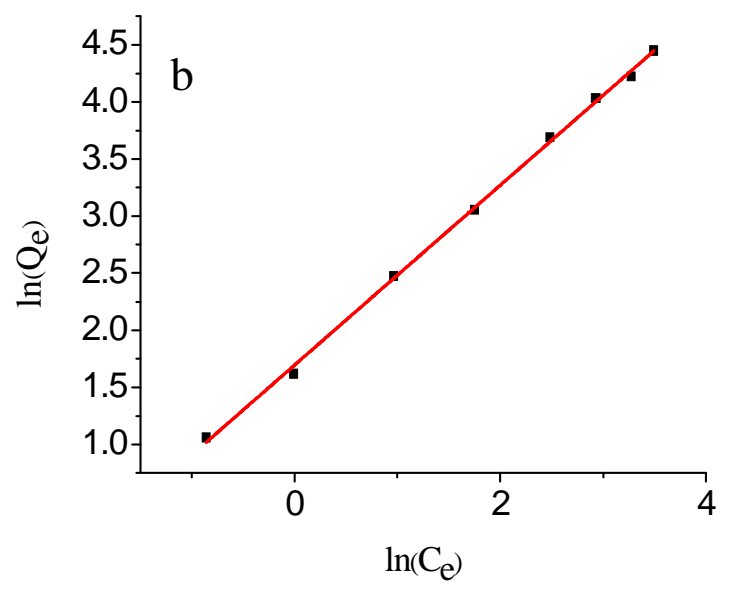

Fig.4 Freundlich isotherm model for atrazine adsorption on MGO.

The effect of contact time on atrazine adsorption with the MGO was investigated using an initial concentration of $50 \mathrm{mg} / \mathrm{L}$. The results (Fig.5) showed that the adsorption occurred rapidly within the first $1 \mathrm{~h}$ and then gradually slowed down until reaching equilibrium $(2 \mathrm{~h})$. The kinetics of atrazine adsorption was studied by using pseudo-first-order and pseudo-second-order kinetic models. It was found that the pseudo-second order kinetic equation could preferably describe the adsorption (Fig.6). 


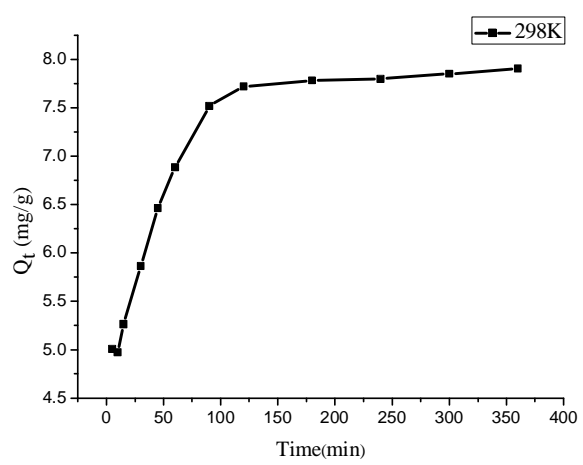

Fig.5 Effect of contact time on atrazine adsorption on MGO.

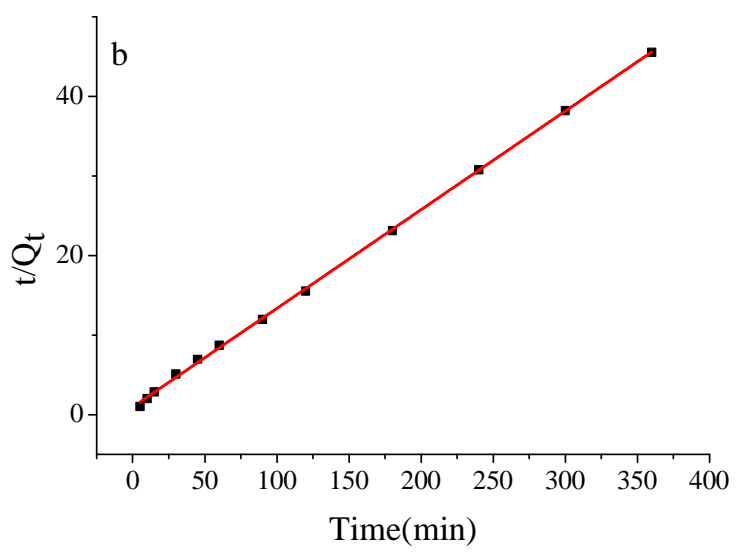

Fig.6 Pseudo-second-order kinetic plots for atrazine adsorption on MGO.

\section{Conclusions}

Magnetic graphene oxide was successfully synthesized and applied to adsorption of atrazine from aqueous solutions. MGO has hexatomic ring as well as carboxyl, carbonyl, epoxy and hydroxy groups, which resulted in $\pi-\pi$ interaction and hydrogen -bond interaction of MGO with atrazine. The adsorption results obtained from this study suggest that the prepared MGO can act as a suitable adsorbent for efficient removal of low concentration of atrazine in industrial effluents.

\section{Acknowledgements}

This work was financially supported by the National Natural Science Foundation of China (No.41272262), the Science and Technology Planning Project of Guangdong Province, China (No. 2014A040401077), and the Major Projects (Natural Science) of Education Department of Guangdong Province, China (No.261555101).

\section{References}

[1] Min G., Wang S. and Zhu H. Sci. Total Environ. 2008, 396(1): 79-85

[2] Zou J., Ma X., Dang Y. and Chen Y. J Anal At Spectrom. 2014; 29(9): 1692-1697.

[3] Chen J., Ma X. and Wu Y. Food Anal Methods. 2014; 7(5): 1083-1089.

[4] Li C., Ma X., Zhang X., et al. J. Sep. Sci., 2017, 40(7): 1621-1628.

[5] Li D., Ma X., Wang R., et al.. Anal. Bioanal. Chem., 2017, 409(5): 1165-1172.

[6] Chen Y., Ma X., Huang M., Peng, J., Li C. Anal Methods. 2016; 8(4): 824-829. 
[7] Marcano, D. C., Kosynkin, D. V., Berlin, J. M., Sinitskii, A., Sun, Z. and Slesarev, A. ACS Nano. 2010, 4, 4806-4814

[8] Chandra, V., Park, J., Chun, Y., Lee, J.W., Hwang, I. C.and Kim, K. S. ACS Nano.2010, 4(7), 3979-3986. 